\title{
Models for Simulation and Control of Underwater Vehicles
}

\author{
Jorge Silva ${ }^{1}$ and João Sousa ${ }^{2}$ \\ ${ }^{1}$ Engineering Institute of Porto \\ ${ }^{2}$ University of Porto \\ Portugal
}

\section{Introduction}

Nowadays, the computational power of the average personal computer provides to a vast audience the possibility of simulating complex models of reality within reasonable time frames. This chapter presents a review of modelling techniques for underwater vehicles with fixed geometry, giving emphasis on their application to real-time or faster than realtime simulation.

In the last decade there was a strong movement towards the development of Autonomous Underwater Vehicles (AUV) and Remotely Operated Vehicles (ROV). These two classes of underwater vehicles are intended to provide researchers with simple, long-range, low-cost, rapid response capability to collect pertinent environmental data. There are numerous applications for AUV and ROV, including underwater structure inspection, oceanographic surveys, operations in hazardous environments, and military applications. In order to fulfil these objectives, the vehicles must be provided with a set of controllers assuring the desired

$\varepsilon$ type of autonomous operation and offering some aid to the operator, for vehicles which can ठ․ be teleoperated.

The design and tuning of controllers requires, on most methodologies, a mathematical model of the system to be controlled. Control of underwater vehicles is no exception to this rule. The most common model in control theory is the classic system of differential equations, where $\mathrm{x}$ and $\mathrm{u}$ are denominated respectively state vector and input vector:

$$
\dot{x}=f(x, u)
$$

§ In this framework, the most realistic models of underwater vehicles require $f(x, u)$ to be a $\widetilde{\widetilde{O}}$ nonlinear function. However, as we will see, under certain assumptions, the linearization of $(x, u)$ may still result in an acceptable model of the system, with the added advantage of the analytic simplicity.

if On the other hand, simulation, or more specifically, numerical simulation, does not require \& a model with the conceptual simplicity of Eq.(1). In this case, we are not concerned with an analytic proof of the system's properties. The main objective is to compute the evolution of a set of state variables, given the system's inputs.

The hydrodynamic effects of underwater motion of a rigid body are well described by the Navier-Stokes equations. However, these equations form a system of nonlinear partial Source: New Approaches in Automation and Robotics, Book edited by: Harald Aschemann, ISBN 978-3-902613-26-4, pp. 392, May 2008, I-Tech Education and Publishing, Vienna, Austria 
differential equations whose solution is very hard to compute for general problems. Computational fluid dynamics deal with the subject of solving these equations. However, even with today's computer technology and software packages, the time to obtain the solution on average computer, even for simple scenarios, is still far from real-time. This kind of simulation may return very accurate results, but the computation times are not acceptable if simulation of long term operation is desired. These models are just too complex for control design and approximations are used for this purpose.

If near, or faster than, real-time simulations are desired, acceptable results can be obtained by simulating the model employed in the control design. Real-time response is most useful when teleoperation simulation is desired. In this case, as in car or flight simulators, the user interacts with the underwater vehicle simulator by setting references or direct actuator commands on a graphical user interface.

Usually we are interested in checking the performance of the vehicle's controllers in a set of operation scenarios. This is done because the control design may involve certain simplifications or heuristic methods which make it difficult to analytically characterize certain parameters of the system's response, such as settling time or peak values (overshoots) during transient phases of operation. Simulation is an important tool for controller tuning and for exposing certain limit situations (e.g., actuator saturation) that may be hard to describe analytically on the model employed for control design.

The typical design cycle involves the test of different control laws and navigation schemes. In most cases, the control system must be replicated in a simulation environment, usually on a different language. Even when that is done correctly, it is difficult to keep consistency between that implementation and the final control system, which may be subject to updates from other sources. It is possible, as described in (Silva et al. 2007), to have a single implementation of the control software to function unmodified in both real-life and simulated environments. Instead of writing separate code first for a prototyping environment and then for the final version, this approach allows the employment of the stable/final software in the overall design cycle. Therefore, the simulation may be seen also as a debugging tool of the overall software design process. Underwater vehicle's mission management, with special regards to autonomous operation, may involve complex logic, besides the continuous control laws. It is of the major importance to test the implementation of that logic, namely switching between manoeuvres, manoeuvre coordination, event detection, etc. Since real-life missions may last for some hours, it is quite useful to simulate these missions in compressed simulated time.

The literature from naval architecture proposes several models for underwater vehicles following the structure of Eq.(1). The main difference between these models is the way how the hydrodynamic phenomena associated with underwater rigid body motion is modelled. The model described in (Healey \& Lienard, 1993) is used in many works. These authors refine a model that can be traced back to 1967 (Gertler \& Hagen, 1967) in order to describe a box shaped AUV. Most recent works use the framework presented on (Fossen, 1994) which, while less descriptive than the one of (Healey \& Lienard, 1993), is more amenable to direct application of tools from nonlinear control. Recent research on underwater vehicle's motion equations can still be found, for instance on (Nahon, 2006).

However, in general, the literature only provides the general equations of the models. These models are parametrized by tens (sometimes over one hundred) of coefficients. Some of these coefficients can be easily computed based on direct physical measurements (mass, 
length, etc.). However, the computation of the coefficients related to hydrodynamic effects is not a straightforward task. When considering new designs, the accurate estimation of some of the coefficients, mainly those associated with hydrodynamic phenomena, usually requires hydrodynamics tests. Although ingenious techniques can be used, see for instance (von Ellenrieder, 2006), these tests are usually expensive or involve an apparatus which is not justifiable for every institution.

Certain software packages can be used to obtain more accurate parameters. For instance, (Irwin \& Chavet, 2007) present a study comparing results obtained with Computational Fluid Dynamics with those of classical heuristic formulas. However, software packages for this purpose are usually expensive, or of limited accessibility.

The simplest alternative approaches rely on empirical formulas, or on adapting the coefficients from well-proved models from similar vehicles. In fact, empirical results show that, for vehicles of the same shape, the hydrodynamic effects can be normalized as a function of scale and vehicle's operating speed. For instance, the work of (Healey \& Lienard, 1993) presents the complete set of numeric parameters for the model proposed by the authors on that work. However, the later method is suitable only for vehicles with similar shape to those whose models are known and the former requires the vehicle being modelled to follow closely the assumptions of the empirical formulas.

In what follows, we review a standard nonlinear model derived from (Fossen, 1994), describe further aspects of the hydrodynamic phenomena, and explain how the symmetries of the vehicle can be explored in order to reduce the number of considered coefficients.

The final conclusions are drawn based on our experiments with the Light Autonomous Underwater Vehicle (LAUV) designed and built at University of Porto (see Fig. 1). LAUV is a torpedo shaped vehicle, with a length of 1.1 meters, a diameter of $15 \mathrm{~cm}$ and a mass of approximately $18 \mathrm{~kg}$. The actuator system is composed of one propeller and 3 or 4 control fins (depending on the vehicle version), all electrically driven. We compare trajectories logged during the operation of the LAUV with trajectories obtained by simulation of the vehicle's mathematical model.

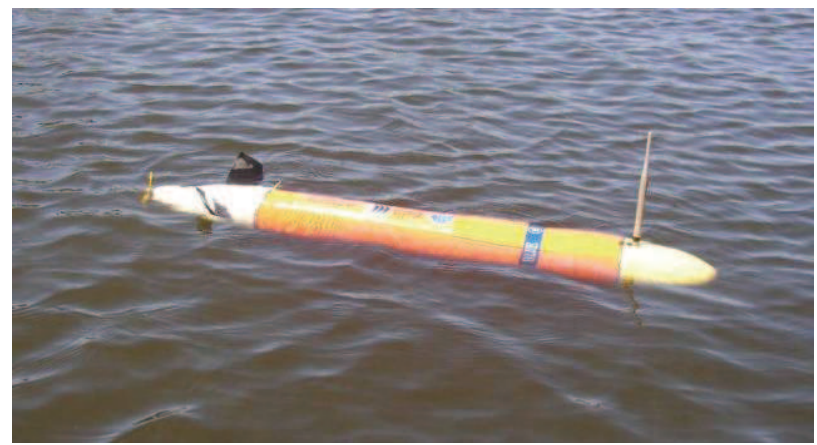

Fig. 1. Light Autonomous Underwater Vehicle (LAUV) designed and built at University of Porto.

\section{Underwater vehicle dynamics}

When discussing underwater vehicle dynamics we typically consider two coordinate frames: the Earth-Fixed Frame and the Body-Fixed Frame. 
The Earth-Fixed Frame defines a coordinate system with origin fixed to an arbitrary point on the surface of the Earth and following the north-east-down convention: $x$ points due North, $y$ points due East, and $z$ points toward the center of the Earth. For marine applications, this frame is considered the inertial frame.

In the Body-Fixed Reference Frame the origin and axes of the coordinate system are fixed with respect to the (nominal) geometry of the vehicle. The orientation of the axes is as shown on Fig. 2: if the underwater vehicle has a plane of symmetry (and we will assume here that they all do) then $x_{B}$ and $z_{B}$ lie in that plane of symmetry. $x_{B}$ is chosen to point forward and $z_{B}$ is chosen to point downward. Usually the body axes coincide with the principal axes of inertia of the vehicle. Fig. 2 shows one possibility. The origin of the bodyfixed frame is frequently chosen to coincide with the center of gravity. This is a natural choice given the equations of rigid body motion. However, in many situations, most remarkably during prototyping, the center of mass may be changing relatively to the vehicle's geometry. That makes necessary to recalculate the moments due some of the forces involved on vehicle's motion (e.g., forces due to the actuators). Therefore, in those cases, a more useful choice would be a point relative to the vehicle's shape such as the center of pressure (described later) or simply the geometrical center.

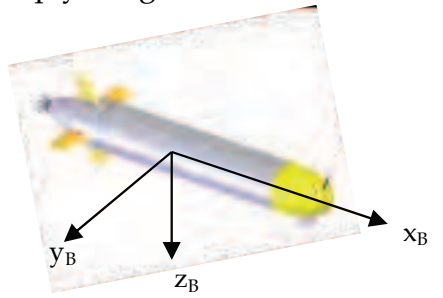

Fig. 2. Body-Fixed Reference Frame.

The minimum set of variables that completely describe the vehicle's position, orientation, and linear and angular velocities is called vehicle state. The most commonly chosen state variables are the inertial position and orientation of the vehicle, and its body-fixed linear and angular velocities. The orientation of the vehicle with respect to inertial space can be described by the Euler angles. These angles are termed yaw angle $(\psi)$, pitch angle $(\theta)$ and roll angle $(\phi)$. This implies three different rotations will be needed (one for each axis). The order in which these rotations are carried out is not arbitrary. The standard coordinate systems and rotations (Lewis, 1989), are as defined in Fig. 3. In what follows, the notation from the Society of Naval Architects and Marine Engineers (SNAME) is used (Lewis, 1989). The motions in the body-fixed frame are described by 6 velocity components $u, v, w, p, q$ and $r$. Let us define the following vectors:

$$
\begin{aligned}
v_{1} & =\left[\begin{array}{lll}
\mathrm{u} & \mathrm{v} & \mathrm{w}
\end{array}\right]^{\mathrm{T}} \\
v_{2} & =\left[\begin{array}{lll}
\mathrm{p} & \mathrm{q} & \mathrm{r}
\end{array}\right]^{\mathrm{T}} \\
v & =\left[\begin{array}{ll}
\mathrm{v}_{1}^{\mathrm{T}} & \mathrm{v}_{2}^{\mathrm{T}}
\end{array}\right]^{\mathrm{T}}
\end{aligned}
$$

The body fixed linear velocities $\mathrm{u}, \mathrm{v}$ and $\mathrm{w}$ are termed, respectively, surge, sway and heave. We adopt the following convention: when considering slow varying ocean currents, these velocities are relative to a coordinate frame moving with the ocean current. 
The vehicle's position and orientation in the inertial frame are defined by the following vectors:

$$
\begin{aligned}
\eta_{1} & =\left[\begin{array}{lll}
x & y & z
\end{array}\right]^{\mathrm{T}} \\
\eta_{2} & =\left[\begin{array}{lll}
\phi & \theta & \psi
\end{array}\right]^{\mathrm{T}} \\
\eta & =\left[\begin{array}{ll}
\eta_{1}^{\mathrm{T}} & \eta_{2}^{\mathrm{T}}
\end{array}\right]^{\mathrm{T}}
\end{aligned}
$$
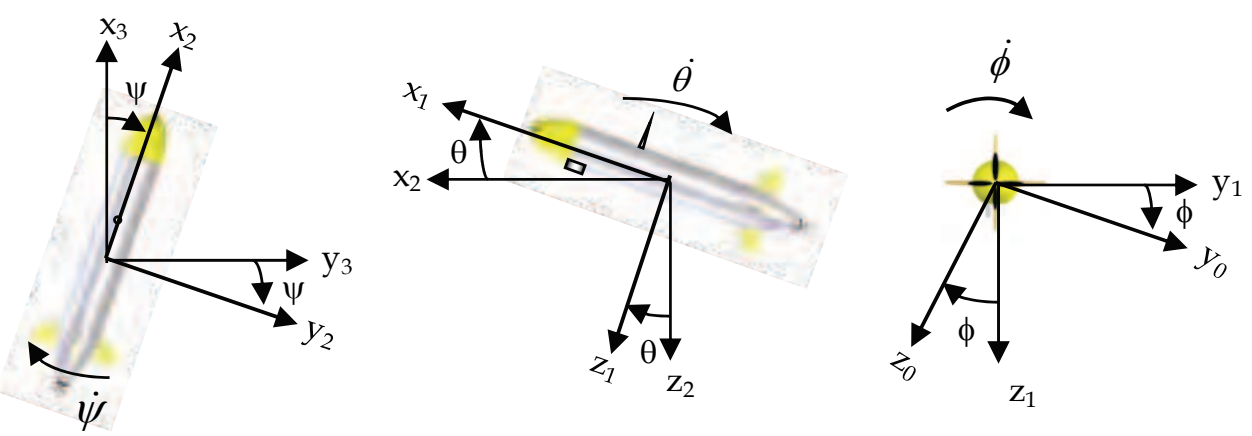

Fig. 3. Euler angles definition: yaw, pitch and roll.

The twelve basic states of an underwater vehicle may therefore be written as (again, this is just one possible choice, but it is the standard one):

\begin{tabular}{|c|l|c|}
\hline Name & \multicolumn{1}{|c|}{ Description } & Unit \\
\hline $\mathrm{u}$ & Linear velocity along body-fixed x-axis (surge) & $\mathrm{m} / \mathrm{s}$ \\
\hline $\mathrm{v}$ & Linear velocity along body-fixed y-axis (sway) & $\mathrm{m} / \mathrm{s}$ \\
\hline $\mathrm{w}$ & Linear velocity along body-fixed z-axis (heave) & $\mathrm{m} / \mathrm{s}$ \\
\hline $\mathrm{p}$ & Angular velocity about body-fixed x-axis & $\mathrm{rad} / \mathrm{s}$ \\
\hline $\mathrm{q}$ & Angular velocity about body-fixed y-axis & $\mathrm{rad} / \mathrm{s}$ \\
\hline $\mathrm{r}$ & Angular velocity about body-fixed z-axis & $\mathrm{rad} / \mathrm{s}$ \\
\hline$\psi$ & Heading angle with respect to the reference axes & $\mathrm{rad}$ \\
\hline$\theta$ & Pitch angle with respect to the reference axes & $\mathrm{rad}$ \\
\hline$\phi$ & Roll angle with respect to the reference axes & $\mathrm{rad}$ \\
\hline $\mathrm{x}$ & Position with respect to the reference axes (North) & $\mathrm{m}$ \\
\hline $\mathrm{y}$ & Position with respect to the reference axes (East) & $\mathrm{m}$ \\
\hline $\mathrm{z}$ & Position with respect to the reference axes (Down) & $\mathrm{m}$ \\
\hline
\end{tabular}

Table 1. Vehicle States

\subsection{Dynamic equations}

The evolution of $\eta$ is defined by the following kinematic equation:

$$
\dot{\eta}=J\left(\eta_{2}\right) v
$$


This equation defines the relationship between the velocities on both reference frames, with the term $\mathrm{J}\left(\eta_{2}\right)$ given as follows:

$$
\begin{gathered}
\mathrm{J}_{1}\left(\eta_{2}\right)=\left[\begin{array}{ccc}
\cos \theta \cos \psi & (-\cos \phi \sin \psi+\sin \phi \sin \theta \cos \psi) & (\sin \phi \sin \psi+\cos \phi \sin \theta \cos \psi) \\
\cos \theta \sin \psi & (\cos \phi \cos \psi+\sin \phi \sin \theta \sin \psi) & (-\sin \phi \cos \psi+\cos \phi \sin \theta \sin \psi) \\
-\sin \theta & \cos \theta \sin \psi & \cos \theta \cos \phi
\end{array}\right] \\
\mathrm{J}_{2}\left(\eta_{2}\right)=\left(\frac{1}{\cos \theta}\right)\left[\begin{array}{ccc}
\cos \theta & \sin \phi \sin \theta & \cos \phi \sin \theta \\
0 & \cos \phi \cos \theta & -\sin \phi \cos \theta \\
0 & \sin \phi & \cos \phi
\end{array}\right] \\
J\left(\eta_{2}\right)=\left[\begin{array}{cc}
J_{1}\left(\eta_{2}\right) & 0 \\
0 & J_{2}\left(\eta_{2}\right)
\end{array}\right]
\end{gathered}
$$

Note that there is a singularity in Eq. (8) when the pitch angle is $\pm 90^{\circ}$. Underwater vehicles typically do not operate close to this singularity. If we want to consider operation in that zone, one possible alternative to the Euler angles is the quaternion representation. For further details see, for instance, (Fossen, 1994).

For most marine control applications it is valid to assume the principle of superposition can be applied when considering environmental disturbances. For underwater vehicles operating far from the surface, the main environmental disturbance to be considered is due to marine currents. Ocean and river currents arise due to a multitude of factors but, in the generality of the operations, they have a low spatial and temporal rate of change with respect to the vehicle dynamics. Therefore we can incorporate the effect of currents in the vehicle model by considering that $v$ is relative to a frame moving with the water current velocity $v_{c}$, where $v_{c}=\left[v_{c x}, v_{c y}, v_{c z}, 0,0,0\right]$ is the earth-fixed current velocity vector. The only change to the previously defined model will be in the kinematic equations which will come as follows:

$$
\dot{\eta}=J\left(\eta_{2}\right) v+v_{c}
$$

The equations of motion are composed of the standard terms for the motion of an ideal rigid body plus the terms due to hydrodynamic forces and moments and the terms due to propulsive forces. The usual approach to model the hydrodynamic terms consists of considering the following effects: restoring forces, the simplest one, which depends only on the vehicle weight, buoyancy and relative positions of the centers of gravity and buoyancy; added mass, which describes the pressure induced forces/moments due to forced harmonic motion of the body; drag, caused by skin friction (either laminar or turbulent) and vortex shedding; and lift, due to the fluid flow whose direction is changed perpendicularly to vehicle's linear velocity vector. As hinted in the introduction, added mass, drag and lift are very hard to describe accurately by theoretical methods. An extensive discussion about the added mass effect is presented in (Brennen, 1982).

The drag effect is modelled as a force opposing to the vehicle's velocity. The lift effect is modelled as a force perpendicular to the vehicle's velocity. Both forces act on the center of 
pressure of the vehicle and are a function of the vehicles' shape and of the square of its velocity. The center of pressure is also strongly dependent on vehicle's shape. For a more indepth analysis of this subject see, for instance, (Hoerner, 1992).

With the exception of the gravity and buoyancy forces, these effects are best described in the Body-Fixed Frame. Therefore, the remaining equations of motion, describing the vehicle's kinetics, can be presented in the following compact form:

$$
\mathrm{M} \dot{v}+\mathrm{C}(v) v+\mathrm{D}(v) v+\mathrm{g}(\eta)=\tau_{\text {act }}
$$

$M$ is the constant inertia and added mass matrix of the vehicle, $C(v)$ is the Coriolis and centripetal matrix, $D(v)$ is the Damping matrix, $g(\eta)$ is the vector of restoring forces and moments and $\tau_{\text {act }}$ is the vector of body-fixed forces from the actuators. We follow the common formulation where the lift and drag terms are both accounted in the damping matrix.

For vehicles with a streamlined shape, theoretical and empirical formulas may be used. However, it must be remarked that in practice these vehicles are not quite as regular as assumed in the formulas usually employed for added mass, drag and lift: they have antennas, transducers and other protuberances that affect those effects, with special incidence on the drag terms. Therefore we should look at the formulas as giving underestimates of the true values of the coefficients.

In certain situations it may be useful to consider the following simplifications: if the vehicle's weight equals its buoyancy and the center of gravity is coincident with the center of buoyancy, $g(\eta)$ is null; for an AUV with port/starboard, top/bottom and fore/aft symmetries, $\mathrm{M}$ and $\mathrm{D}(v)=\mathrm{D}_{1}(v)+\mathrm{D}_{2}(v)$ are diagonal. In the later case, the damping matrix has the following form:

$$
\begin{gathered}
\mathrm{D}_{1}(v)=\operatorname{diag}\left(\mathrm{X}_{\mathrm{u}}, \mathrm{Y}_{\mathrm{v}}, \mathrm{Z}_{\mathrm{w}}, \mathrm{K}_{\mathrm{p}}, \mathrm{M}_{\mathrm{q}}, \mathrm{M}_{\mathrm{q}}\right) \\
\mathrm{D}_{2}(\mathrm{v})=\operatorname{diag}\left(\mathrm{X}_{\mathrm{u}|u|}|\mathrm{u}|, \mathrm{Y}_{\mathrm{v}|\mathrm{v}|}|\mathrm{v}|, \mathrm{Z}_{\mathrm{w}|\mathrm{w}|}|\mathrm{w}|, \mathrm{K}_{\mathrm{p}|\mathrm{p}|}|\mathrm{p}|, \mathrm{M}_{\mathrm{q}|q|}|\mathrm{q}|, \mathrm{N}_{\mathrm{r}|\mathrm{r}|}|\mathrm{r}|\right)
\end{gathered}
$$

For low velocities, the quadratic terms on Eq. 13, such as $Y_{\mathrm{v}|\mathrm{v}|}|\mathrm{v}|$, may be considered negligible. However, in practice, the fore/aft symmetry is rarely verified and non-diagonal terms should be considered. Even so, certain simplifications can be further considered. For instance, in torpedo shaped vehicles, some of the coefficients affecting the motion on the vertical plane are the same as those affecting the motion on the horizontal plane, reducing the number of different coefficients that must be estimated.

Some of the models found in the literature, e.g. (Prestero 2001; Leonard \& Graver, 2001; Conte \& Serrani, 1996; Ridley et al., 2003), do not consider the linear damping terms contained on $\mathrm{D}_{1}(\mathrm{v})$. These terms may play an important role in the design of the control system, namely on local stability analysis. For low velocities scenarios the quadratic damping terms become very small. If the linear damping is ignored, the linearization of the system model around the equilibrium point may falsely reveal a locally unstable system. This leads the control system designer to counteract by adding linear damping in the form of velocity feedback, which potentially could be unnecessary, leading to conservative designs. In fact, it is possible to find examples in the literature where the authors perform a worst case analysis, by totally disregarding the damping matrix (Leonard 1996; Chyba 2003). 


\subsection{Actuators}

In the last years there has been a trend in the research of biologically inspired actuators for underwater vehicles, see for instance (Tangorra, 2007). The development of vehicles employing variable buoyancy and center of mass (e.g., gliders) is also underway (Bachmayer, 2004). However, the preferred types of actuators for small size AUVs still are electrically driven propellers and fins, due to its simplicity, robustness and low cost.

When high manoeuvrability is desired, full actuation is employed (for instance, with two longitudinal thrusters, two lateral thrusters and two vertical thrusters). For over-actuated vehicles, thruster allocation schemes may be applied in order to optimize performance and power consumption. However, for a broad range of applications the cost effectiveness of under-actuated vehicles is still a factor of preference. In those cases, a smaller number of thrusters, eventually coupled with fins, is employed. This approach is applied in most torpedo-like AUVs: there is a propeller for actuation in the longitudinal direction and fins for lateral and vertical actuation. In this case, $\tau_{a c t}$ depends only on 3 parameters: propeller velocity, horizontal fin inclination and vertical fin inclination.

Dynamic models for propellers can be found in (Fossen, 1994) and this is still an active area of research (D'Epagnier, 2006). However, the dynamics of the thruster motor and fin servos are generally faster than the remaining dynamics. Therefore, they can be frequently excluded from the model, namely when operation at steady speed is considered as opposed to dynamic positioning, or station keeping.

\subsection{Simplified models}

For a large class of underwater vehicles it is usual to consider decoupled modes of operation, see for instance (Healey \& Lienard, 1993), the most common being motion on the horizontal plane, involving changes on $\mathrm{x}, \mathrm{y}, \psi, \mathrm{v}$, and motion on the vertical plane aligned with the body fixed $x-z$ axes, involving changes on $z, \theta, w$ and $q$. In the later mode, assuming small deviations from 0 on the pitch angle, a linearized model can be used without introducing significant error (the $\mathrm{a}_{\mathrm{ij}}, \mathrm{k}_{\mathrm{w}}$ and $\mathrm{k}_{\mathrm{q}}$ coefficients can be calculated as a function of the coefficients of the full nonlinear model):

$$
\left[\begin{array}{c}
\dot{\mathrm{z}} \\
\dot{\theta} \\
\dot{\mathrm{w}} \\
\dot{\mathrm{q}}
\end{array}\right]=\left[\begin{array}{cccc}
0 & -\mathrm{u} & 1 & 0 \\
0 & 0 & 0 & 1 \\
0 & \mathrm{a}_{32} & \mathrm{a}_{33} & \mathrm{a}_{34} \\
0 & \mathrm{a}_{42} & \mathrm{a}_{43} & \mathrm{a}_{44}
\end{array}\right]\left[\begin{array}{c}
\mathrm{z} \\
\theta \\
\mathrm{w} \\
\mathrm{q}
\end{array}\right]+\left[\begin{array}{c}
\mathrm{v}_{\mathrm{cz}} \\
0 \\
\mathrm{k}_{\mathrm{w}} \tau_{\mathrm{w}} \\
\mathrm{k}_{\mathrm{q}} \tau_{\mathrm{q}}
\end{array}\right]
$$

For the purpose path planning on the horizontal plane with piecewise continuous velocity, a simple kinematic model can be used:

$$
\left\{\begin{array}{c}
\dot{\mathrm{x}}=\mathrm{u} \cos (\psi)-\mathrm{v} \sin (\psi)+\mathrm{v}_{\mathrm{cx}} \\
\dot{\mathrm{y}}=\mathrm{v} \cos (\psi)+\mathrm{u} \sin (\psi)+\mathrm{v}_{\mathrm{cx}} \\
\dot{\psi}=\mathrm{r}
\end{array}\right.
$$

For the purpose of path planning, it is considered that the actuators produce the desired velocities instantaneously. The allowable ranges for $\mathrm{u}, \mathrm{v}$ and $\mathrm{r}$ must be the same as the ones 
verified for the full dynamic model, or measured in real operation. While this model introduces some errors that must be compensated later by the on-line control system, this is very useful for the general path planning algorithms. If the vehicle does not possess lateral actuation, such as a torpedo, the model drops the terms on $\mathrm{v}$ and becomes the well-known unicycle model.

\section{Results and discussion}

In (Silva et al., 2007) we describe a simulation environment which allows us to simulate AUV operation in real-time and with direct interaction with the control software. All software was written in $\mathrm{C}++$ and is based on the Dune framework, also developed at the University of Porto. Using this framework, the control software and simulation engine may run either on a desktop computer or on the final target computer. Our results show that realistic real-time and faster than real-time simulation of underwater vehicles is quite feasible in today's computers. The trajectories obtained with the exact same inputs as those used in experiments in the water differ slightly from the real trajectories. However, in what concerns simulation of closed-loop operation, the feedback employed on the control laws smoothes out the effects of parameter uncertainty. Therefore it is possible to observe a good correlation between the performance of the controlled system in simulation and that obtained in real operation. This conclusion is drawn using the exact same controllers and timings on simulation and real operation. This result is not as assuring as a complete analytical proof but, then again, none of the currently employed models are perfect descriptions of the reality therefore, even an analytical study does not guarantee the planned behaviour when the respective implementation comes to real life operation.

The available methods are quite satisfactory for high level mission planning and already provide a good basis for initial controller tuning. However, additional tuning is still required when it comes to real life vehicle operation. Research on models whose simulation can be done in reasonable time while providing an increasing level of adherence to reality should continue.

\section{References}

Bachmayer, R.; Leonard, N.E.; Graver, J.; Fiorelli, E.; Bhatta, P. \& Paley, D. (2004). Underwater gliders: recent developments and future applications, Proceedings of the 2004 International Symposium on Underwater Technology, pp. 195-200, Taipei, Taiwan, April 2004

Brennen, C.E (1982). A review of added mass and fluid internal forces, Naval Civil engineering laboratory, California

Chyba, M.; Leonard, N. E. \& Sontag, E. (2003). Singular trajectories in multi-input timeoptimal problems: Application to controlled mechanical systems, Journal of Dynamical and Control Systems, Vol. 9, No. 1, pp. 73-88

Conte, G. \& Serrani, A. (1996) Modelling and simulation of underwater vehicles, Proceedings of the 1996 IEEE International Symposium on Computer-Aided Control System Design, pp. 62-67, Dearborn, Michigan, September 1996

D'Epagnier, K. P. (2006). AUV Propellers: Optimal Design and Improving Existing Propellers for Greater Efficiency, Proceedings of the OCEANS 2006 MTS/IEEE Conference, Boston, Massachusetts USA, September 2006 
Fossen, T.I. (1994). Guidance and Control of Ocean Vehicles, John Wiley and Sons, Inc., New York

Gertler, M. \& Hagen, G. R. (1967). Standard equations of motion for submarine simulation, Naval Ship Research and Development Center, Report 2510.

Healey, A. J. \& Lienard, D. (1993). Multivariable Sliding Mode Control for Autonomous Diving and Steering of Unmanned Underwater Vehicles, IEEE Journal of Oceanic Engineering, Vol. 18, No. 3, pp. 1-13

Leonard, N. E. (1996). Stabilization of steady motions of an underwater vehicle, Proceedings of the 1996 IEEE Conference on Decision and Control, pp. 961-966, Kobe, Japan, December 1996

Leonard, N. E. \& Graver, J. G. (2001). Model-based feedback control of autonomous underwater gliders, IEEE Journal of Oceanic Engineering (Special Issue on Autonomous Ocean-Sampling Networks), Vol. 26, No. 4, pp. 633-645

Lewis, E. (Ed.) (1989). Principles of Naval Architecture (2nd revision), Society of Naval Architects and Marine Engineers, Jersey City, New Jersey

Hoerner, S. F. \& Borst H. V. (1992). Fluid Dynamic Lift (second edition), published by author, ISBN 9998831636

Irwin, R. P. \& Chauvet, C. (2007). Quantifying Hydrodynamic Coefficients of Complex Structures, Proceedings of the IEEE/OES OCEANS 2007 - Europe, pp. 1-5, Aberdeen, Scotland, June 2007

Nahon, M. (2006). A Simplified Dynamics Model for Autonomous Underwater Vehicles, Journal of Ocean Technology, Vol. 1, No. 1, pp. 57-68

Prestero, T. J. (2001). Development of a six-degree of freedom simulation model for the remus autonomous underwater vehicle, Proceedings of the OCEANS 2001 MTS/IEEE Conference and Exhibition, pp. 450-455, Honolulu, Hawaii, November 2001

Ridley, P.; Fontan, J. \& Corke, P. (2003). Submarine dynamic modelling, Proceedings of the Australian Conference on Robotics and Automation, Brisbane, Australia, December 2003

Silva, J.; Terra, B.; Martins R. \& Sousa, J. (2007). Modeling and Simulation of the LAUV Autonomous Underwater Vehicle, Proceedings of the 13th IEEE IFAC International Conference on Methods and Models in Automation and Robotics, pp. 713-718, Szczecin, Poland, August 2007

Tangorra, J. L.; Davidson, S. N.; Hunter, I. W.; Madden, P. G. A.; Lauder, G. V.; Dong, H.; Bozkurttas, M. \& Mittal, R. (2007). The Development of a Biologically Inspired Propulsor for Unmanned Underwater Vehicles, IEEE Journal of Oceanic Engineering, Vol. 32, No. 3, pp. 533-550

von Ellenrieder, K. D. \& Ackermann, L. E. J. (2006). Force/flow measurements on a lowspeed, vectored-thruster propelled UUV," Proceedings of the OCEANS 2006 MTS/IEEE Conference, Boston, Massachusetts USA, September 2006 


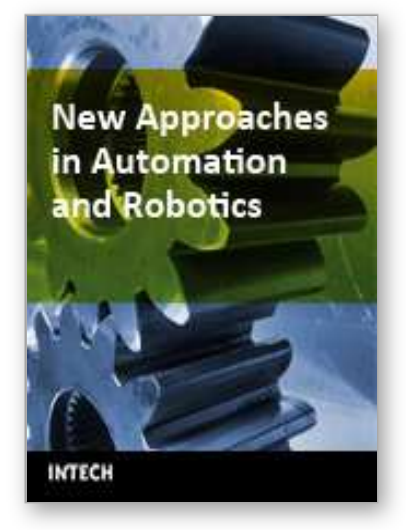

\author{
New Approaches in Automation and Robotics \\ Edited by Harald Aschemann
}

ISBN 978-3-902613-26-4

Hard cover, 392 pages

Publisher I-Tech Education and Publishing

Published online 01, May, 2008

Published in print edition May, 2008

The book New Approaches in Automation and Robotics offers in 22 chapters a collection of recent developments in automation, robotics as well as control theory. It is dedicated to researchers in science and industry, students, and practicing engineers, who wish to update and enhance their knowledge on modern methods and innovative applications. The authors and editor of this book wish to motivate people, especially under-graduate students, to get involved with the interesting field of robotics and mechatronics. We hope that the ideas and concepts presented in this book are useful for your own work and could contribute to problem solving in similar applications as well. It is clear, however, that the wide area of automation and robotics can only be highlighted at several spots but not completely covered by a single book.

\title{
How to reference
}

In order to correctly reference this scholarly work, feel free to copy and paste the following:

Jorge Silva and Joao Sousa (2008). Models for Simulation and Control of Underwater Vehicles, New Approaches in Automation and Robotics, Harald Aschemann (Ed.), ISBN: 978-3-902613-26-4, InTech, Available from:

http://www.intechopen.com/books/new_approaches_in_automation_and_robotics/models_for_simulation_and _control_of_underwater_vehicles

\section{INTECH}

open science | open minds

\author{
InTech Europe \\ University Campus STeP Ri \\ Slavka Krautzeka 83/A \\ 51000 Rijeka, Croatia \\ Phone: +385 (51) 770447 \\ Fax: +385 (51) 686166 \\ www.intechopen.com
}

\author{
InTech China \\ Unit 405, Office Block, Hotel Equatorial Shanghai \\ No.65, Yan An Road (West), Shanghai, 200040, China \\ 中国上海市延安西路65号上海国际贵都大饭店办公楼 405 单元 \\ Phone: +86-21-62489820 \\ Fax: $+86-21-62489821$
}


(C) 2008 The Author(s). Licensee IntechOpen. This chapter is distributed under the terms of the Creative Commons Attribution-NonCommercialShareAlike-3.0 License, which permits use, distribution and reproduction for non-commercial purposes, provided the original is properly cited and derivative works building on this content are distributed under the same license. 